\title{
Two-year cross-sectional studies reveal that single, young MSMs in Shenzhen, China are at high risk for HIV infection
}

Dijing Jia ${ }^{1 \dagger}$, Jin Zhao ${ }^{2 \dagger}$, Yongjian Liu', Xiaolin Wang ${ }^{1}$, Lei Jia ${ }^{1}$, Tao Gui', Lin Chen², Chenli Zheng², Jingwan Han', Tianyi $\mathrm{Li}^{1}$, Jingyun $\mathrm{Li}^{1}$, Hanping $\mathrm{Li}^{1 *}$ and $\mathrm{Lin} \mathrm{Li}^{{ }^{*}}$

\begin{abstract}
Background: Shenzhen City is a rapidly growing area with a large number of floating populations, thus making it difficult to control HIV. Serial cross-sectional studies are helpful for the prediction of epidemiological tendency. In this study, two parallel cross-sectional studies were compared to explore changes in HIV epidemiology in Shenzhen, China.

Methods: Two hundred and fifty newly reported HIV-positive cases were randomly selected in Shenzhen City in 2013 and 2015. Socio-demographical information was collected with informed consent. Full-length gag and partial pol genes were amplified using nested RT-PCR followed by sequencing and phylogenetic analysis. The genotypes of anti-HIV drug resistance were also analyzed. The characteristics of the HIV epidemics of 2013 and 2015 were compared to identify patterns.

Results: The proportion of single, young MSMs dramatically increased in 2015 compared to 2013. Many subtypes, including CRF07_BC (36.4\%), CRF01_AE (34.1\%), CRF55_01B (10.2\%), B (6.4\%), CRF08_BC (3.4\%), CRF59_01B (0.9\%), C (0.7\%), D (0.2\%), CRF68_01B (0.2\%), CRF67_01B (0.2\%), and unique recombinant forms (URFs, 7.3\%), were identified. Close phylogenetic relationships between strains prevalent in Shenzhen and other areas of China was observed. No epidemic cluster confined to single, young MSMs was identified. 0.4 and $2.8 \%$ of the strains contained transmitted drug-resistant mutations in 2013 and 2015, respectively.

Conclusion: Although the interval period is short, changes in HIV epidemiology in Shenzhen City are distinct. Frequent surveillance of HIV epidemics in Shenzhen City is thus necessary. Single, young MSMs have become a high-risk population for HIV infection and should be considered as focus population for HIV prevention and behavior intervention in Shenzhen City.
\end{abstract}

Keywords: HIV-1, Cross-sectional study, Phylogenetic analysis, Drug-resistance

\section{Background}

HIV epidemics have recently undergone a rapid shift in transmission profile in China. High-risk populations of HIV infection have changed several times from the initial injecting drug users (IDUs), former plasma donors, sexual workers, MSMs, to the general population [1]. Together with the shift in predominant transmission

\footnotetext{
*Correspondence: hanpingline@163.com; dearwood@sina.com

${ }^{+}$Dijing Jia and Jin Zhao contributed equally to this work.

'Department of AIDS Research, State Key Laboratory of Pathogen and Biosecurity, Beijing Institute of Microbiology and Epidemiology, Beijing 100071, China

Full list of author information is available at the end of the article
}

routes, major HIV subtypes in China have also changed from subtype Thai B, subtype C, CRF07_BC, and CRF08_BC to CRF01_AE [2]. Considering that HIV epidemics in China continue to evolve and thus it is important to improve its surveillance.

Shenzhen, which is located in Guangdong Province in southern China, is a rapidly growing area with a population of approximately 13.8 million. More than $80 \%$ of the populations of Shenzhen are 'non-local residents' who have household registrations in other regions. Hence, Shenzhen is a city with large-scale migrations of 'floating' individuals, which has become a major challenge in the

(c) The Author(s). 2019 Open Access This article is distributed under the terms of the Creative Commons Attribution 4.0 International License (http://creativecommons.org/licenses/by/4.0/), which permits unrestricted use, distribution, and 
prevention and control of HIV epidemics in China [3-6]. The migration of the floating populations always makes the intervention strategies for these groups ineffective [7, 8]. In recent years, the overall HIV prevalence among these migrants throughout China has been rapidly increasing $[9,10]$. Another big problem caused by 'floating' populations is that they are often associated with local epidemics $[9,11]$ and changes in the distribution of circulating HIV-1 subtypes [12]. Therefore, it is necessary to characterize HIV epidemics in Shenzhen City.

Phylogenetic analysis, in combination with traditional epidemiological surveillance, is useful for describing the transmission dynamics among different populations [13-18]. Distinguishing high-risk populations and understanding the spreading dynamic of HIV strains among different populations would be meaningful for prevention. Cohort studies are a good method for serial surveillance; however, large populations are not feasible for cohort studies due to their related high cost and uncontrollable loss ratio at follow-up. Serial cross-sectional studies are helpful for the prediction of epidemiological trends. Furthermore, changes in HIV high-risk populations can be distinguished, which will be important for HIV prevention and behavior intervention. This study aimed to identify changes in the molecular epidemiology of HIV in Shenzhen City to facilitate the development of preventive strategies.

\section{Materials and methods}

\section{Study subjects and specimens}

This study was approved by the Ethical Review Board, Science and Technology Supervisory Committee of the Beijing Institute of Microbiology and Epidemiology. All of the newly reported HIV-positive and treatment-naïve cases identified in 2013 and 2015 in Shenzhen City were collected by the Shenzhen Center of Disease Control and Prevention. Two hundred and fifty cases were randomly sampled using the simple-random sampling method and enrolled into this study with informed consent. Epidemiologic background information was collected using specific questionnaires by trained interviewers. $10 \mathrm{~mL}$ of peripheral whole blood was obtained and the plasma was separated for extraction of viral RNA.

\section{HIV-1 RNA extraction, amplification, and sequencing}

Viral RNA was extracted from $500 \mu \mathrm{L}$ HIV-1 positive plasma specimens after being concentrated using a High Pure Viral RNA kit (Roche, USA). Viral full-length gag (nucleotides 790-2292 using HXB2 as calibrator) and partial pol genes encompassing the entire protease and partial reverse transcriptase regions (nucleotides 2085-5096 using HXB2 as calibrator) were amplified separately using reverse transcriptional nested PCR as previously described [19]. Briefly, the first round of PCR was fulfilled using a TaKaRa one-step RT-PCR kit, and the second round PCR using High Fidelity Taq (Invitrogen, USA) with primers and thermal cycling conditions as described elsewhere $[19,20]$. Positive PCR products were subjected to Sanger sequencing after purification with a variety of internal specific primers (available on request).

\section{Editing, assembly, genotyping, and phylogenetic analysis of HIV-1 sequences}

Potential contamination was excluded by comparing all sequence segments with all of the known sequences in the HIV database by BLAST search (http://hiv-web.lanl. gov/content/index). All of the sequenced fragments were amended, and gag and pol genes from the same patient were assembled based on the overlapping sequences. In the current study, bidirectional sequencing was performed, and the background noise was very clear/minimal. When a secondary peak of $<30 \%$ was observed, this peak was excluded. Once the secondary peak was $>$ $30 \%$, we considered this position as an ambiguous base. Finally, the consensus sequences from one patient were obtained. We were very cautious on the identification of URFs. No breakpoints within URFs were identified at the joint sites of the gag and pol genes. Finally, the consensus sequence per patient was obtained. HIV sequence quality was determined using an online Quality-Control software (https://www.hiv.lanl.gov/content/sequence/ QC/index.html). HIV genotype was determined by submitting genes to the NCBI viral genotyping tool (http:// www.ncbi.nih.gov/projects/genotyping/formpage.cgi).

HIV genotypes were determined by combining the results of the two regions and further confirmed by phylogenetic analysis. For phylogenetic analysis, all our assembled gag and pol sequences were aligned to the reference sequences separately using MSCLE software.. The final alignment was checked visually and edited manually using the BioEdit software package (version 7.0.0; T. Hall, North Carolina State University, Raleigh, NC, USA). Evolutionary distances were computed using the Kimura 2 parameter method, including both transitions and transversions. Phylogenetic trees were generated using the neighbor-joining method with MEGA6.06 software package and maximum likelihood methods with PhyML3.0 software package. Detailed information on the references used in the current work is provided in Additional file 1: Table S1. The reliability of topologies was estimated by performing bootstrap analysis with 1000 replicates. Possible intertype recombination events were further proven using the online Recombination Identification Program (RIP; version 3.0; http://hiv-web. 
lanl.gov) and confirmed by jpHMM online software (http://jphmm.gobics.de/). The gene sequences were deposited to GenBank as accession numbers MH635658 MH636002, MH632305 - MH632713.

\section{Drug resistance analysis}

All of the assembled partial pol genes were submitted to the Stanford HIV Drug Resistance Database website. TDR mutations were identified using the WHO 2009 list of mutations for surveillance of TDR as implemented in the Calibrated Population Resistance tool (v5.0 beta) (http://hivdb.stanford.edu) [21].

\section{Statistical analysis}

All of the data were double entered into Microsoft Excel 2007 (Microsoft; Redmond, WA, USA). Categorical variables were compared using GraphPad Prism 6 software package. For contingency tables containing more than two rows, rows with both items $<5 \%$ were merged before performing the chi-square test. For contingency tables containing two rows, Fisher's exact test was performed.

\section{Results}

Socio-demographic characteristics of the participants

Two hundred and fifty newly reported HIV-positive participants were enrolled in 2013 and 2015 separately. All of the subjects were not registered permanent residents of Shenzhen City. The geographic distribution of these HIV-positive 'floating' migrants are depicted in Fig. 1. The socio-demographic characteristics of 500 participants are summarized in Table 1 . The median age of the participants was 35.1 years old in 2013 and 33.4 years old in 2015 separately (range: 19-57 years old in 2013 and 18-75 years old in 2015). Distinct shifts found in age distribution, transmission routes, and marital status were observed in the study cohort $(P<0.05)$. A higher number of young $(<30$ years is defined as Age-Young and $\geq 30$ years is defined as Age-Old) individuals were identified in 2015 compared to $2013(P<0.0001)$. In 2013, heterosexual transmission was the predominant route of HIV transmission. However, more MSMs than heterosexually transmitted HIV-positive individuals were identified in $2015(P=0.0174)$. In terms of marital status, the proportion of single individuals significantly increased in $2015(P=0.0017)$. Further detailed analysis showed that the proportion of single, young MSMs

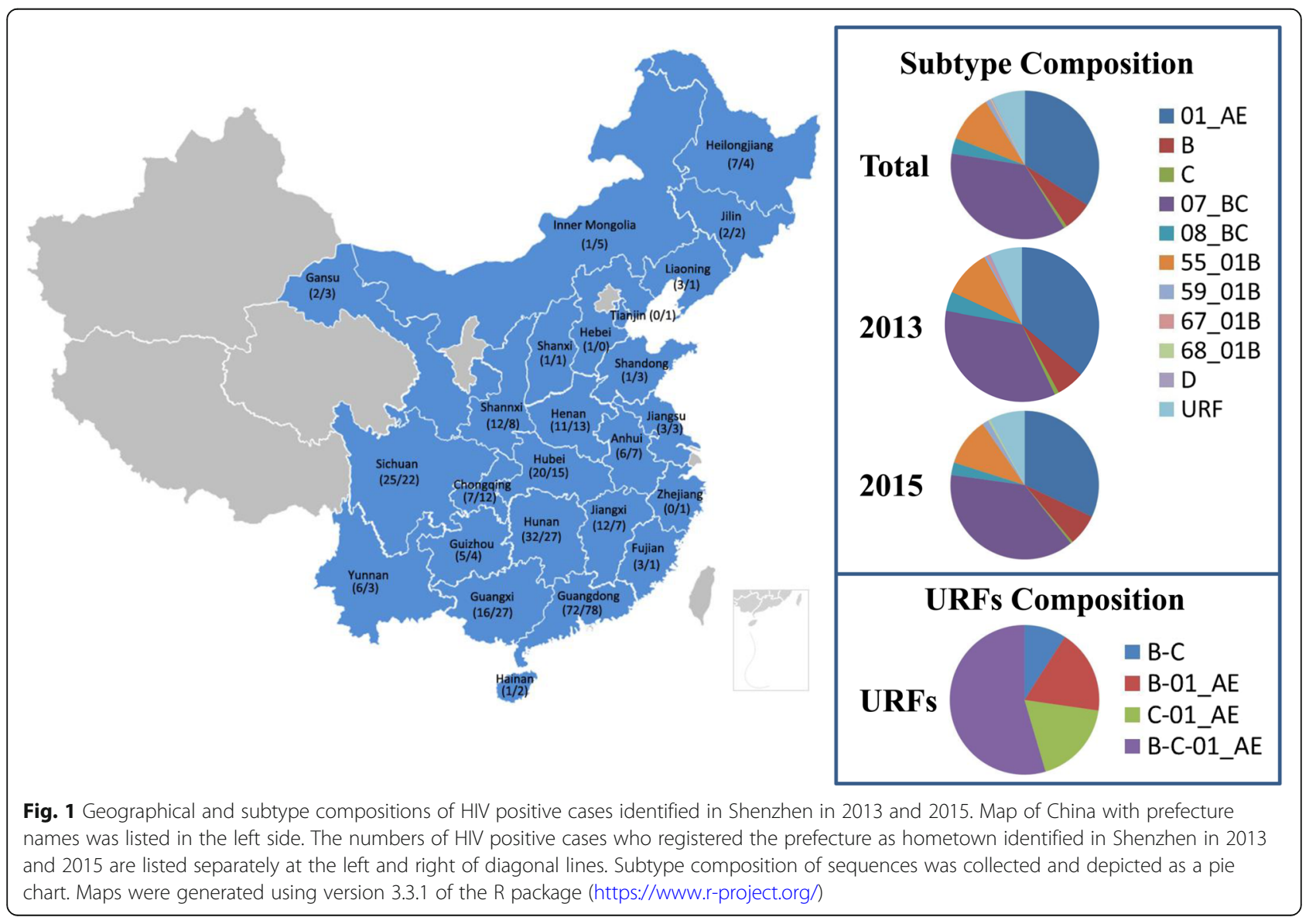


Table 1 Social-demographic characteristics of participants

\begin{tabular}{|c|c|c|c|}
\hline \multirow[t]{2}{*}{ Characteristics } & \multicolumn{2}{|c|}{ Case number (\%) } & \multirow[b]{2}{*}{$P$-value } \\
\hline & 2013 & 2015 & \\
\hline Age (years) & & & 0.001 \\
\hline $2-14$ & $0(0.0 \%)$ & $0(0.0 \%)$ & \\
\hline $15-19$ & $1(0.4 \%)$ & $8(3.2 \%)$ & \\
\hline $20-25$ & $36(14.4 \%)$ & $52(20.8 \%)$ & \\
\hline $26-29$ & $41(16.4 \%)$ & $63(25.2 \%)$ & \\
\hline $30-34$ & $65(26.0 \%)$ & $44(17.6 \%)$ & \\
\hline $35-39$ & $40(16.0 \%)$ & $22(8.8 \%)$ & \\
\hline $40-49$ & $48(19.2 \%)$ & $41(16.4 \%)$ & \\
\hline$\geq 50$ & $18(7.2 \%)$ & $20(8.0 \%)$ & \\
\hline Unknown & $1(0.4 \%)$ & $0(0.0 \%)$ & \\
\hline Gender & & & $>0.05$ \\
\hline Male & $222(88.8 \%)$ & $221(88.4 \%)$ & \\
\hline Female & $27(10.8 \%)$ & $29(11.6 \%)$ & \\
\hline Unknown & $1(0.4 \%)$ & $0(0.0 \%)$ & \\
\hline Ethnic & & & $>0.05$ \\
\hline Han ethnic & $241(96.4 \%)$ & $234(93.6 \%)$ & \\
\hline Minor ethnic & $9(3.6 \%)$ & $16(6.4 \%)$ & \\
\hline Marital status & & & 0.002 \\
\hline Single & $139(55.6 \%)$ & $161(64.4 \%)$ & \\
\hline Married & $88(35.2 \%)$ & $53(21.2 \%)$ & \\
\hline Divorced, separated or widowed & $23(9.2 \%)$ & $33(13.2 \%)$ & \\
\hline Unknown & $0(0 \%)$ & $3(1.2 \%)$ & \\
\hline Education & & & $>0.05$ \\
\hline College or higher & $59(23.6 \%)$ & $76(30.4 \%)$ & \\
\hline Senior high school & 77 (30.8\%) & $62(24.8 \%)$ & \\
\hline Junior high school & $92(36.8 \%)$ & $93(37.2 \%)$ & \\
\hline Primary school or lower & $22(8.8 \%)$ & $19(7.6 \%)$ & \\
\hline Occupation & & & $>0.05^{a}$ \\
\hline Catering services & $6(2.4 \%)$ & $3(1.2 \%)$ & \\
\hline Official & $7(2.8 \%)$ & $7(2.8 \%)$ & \\
\hline Worker & $70(28 \%)$ & $65(26 \%)$ & \\
\hline Commercial service & $61(24.4 \%)$ & $69(27.6 \%)$ & \\
\hline Unemployed & $61(24.4 \%)$ & $78(31.2 \%)$ & \\
\hline Retired & $3(1.2 \%)$ & $3(1.2 \%)$ & \\
\hline Migrant laborer & $2(0.8 \%)$ & $4(1.6 \%)$ & \\
\hline Student & $1(1.6 \%)$ & $2(0.8 \%)$ & \\
\hline Others & $4(0.4 \%)$ & $12(4.8 \%)$ & \\
\hline Unknown & 35 (14.0\%) & $7(2.8 \%)$ & \\
\hline Route of transmission & & & 0.017 \\
\hline Heterosexual & $133(53.2 \%)$ & $112(44.8 \%)$ & \\
\hline MSM & $101(40.4 \%)$ & $130(52.0 \%)$ & \\
\hline IDU & $12(4.8 \%)$ & $4(1.6 \%)$ & \\
\hline Blood-borne & $1(0.4 \%)$ & $1(0.4 \%)$ & \\
\hline Unknown & $3(1.2 \%)$ & $3(1.2 \%)$ & \\
\hline
\end{tabular}

${ }^{a}$ Note: Unknown group was omitted before analysis dramatically increased from $14.0 \%$ in 2013 to $26.4 \%$ in 2015. $(P<0.01)$ (Table 2$)$.

\section{Distribution of HIV subtypes}

A total of 345 full-length $g a g$ genes (69.0\%) and 410 partial pol genes $(82.0 \%)$ were successfully obtained from 500 samples. No evidence of sample contamination was found. Quality control of sequences showed that all of the gene structures were normal and with the correct open reading frames (ORFs). The subtypes of the HIV

Table 2 Distribution of participants based on age, marital status and transmission route

\begin{tabular}{|c|c|c|}
\hline \multirow[t]{2}{*}{ Characteristics } & \multicolumn{2}{|c|}{ Case number (\%) } \\
\hline & 2013 & 2015 \\
\hline Age-Young ( $<30$ years old $)$ & $79(31.6 \%)$ & $123(49.2 \%)$ \\
\hline Transmission route (MSM) & $36(14.4 \%)$ & $72(28.8 \%)$ \\
\hline Marital status (Single) & $35(14.0 \%)$ & $66(26.4 \%)$ \\
\hline Marital status (Married) & $1(0.4 \%)$ & $6(2.4 \%)$ \\
\hline Marital status (DSW $\left.{ }^{a}\right)$ & $0(0.0 \%)$ & $0(0.0 \%)$ \\
\hline Marital status (Unknown) & $0(0.0 \%)$ & $0(0.0 \%)$ \\
\hline Transmission route (Heterosexual) & $42(16.8 \%)$ & $46(18.4 \%)$ \\
\hline Marital status (Single) & $33(13.2 \%)$ & 39 (15.6\%) \\
\hline Marital status (Married) & $9(3.6 \%)$ & $6(2.4 \%)$ \\
\hline Marital status (DSWª) & $0(0.0 \%)$ & $1(0.4 \%)$ \\
\hline Marital status (Unknown) & $0(0.0 \%)$ & $0(0.0 \%)$ \\
\hline Transmission route (Others) & $1(0.4 \%)$ & $3(1.2 \%)$ \\
\hline Marital status (Single) & $1(0.4 \%)$ & $3(1.2 \%)$ \\
\hline Marital status (Married) & $0(0.0 \%)$ & $0(0.0 \%)$ \\
\hline Marital status (DSW $\left.{ }^{a}\right)$ & $0(0.0 \%)$ & $0(0.0 \%)$ \\
\hline Marital status (Unknown) & $0(0.0 \%)$ & $0(0.0 \%)$ \\
\hline Age-Old ( $\geq 30$ years old) & $171(68.4 \%)$ & $127(50.8 \%)$ \\
\hline Transmission route (MSM) & $65(26.0 \%)$ & $58(23.2 \%)$ \\
\hline Marital status (Single) & $42(16.8 \%)$ & $39(15.6 \%)$ \\
\hline Marital status (Married) & $18(7.2 \%)$ & $12(4.8 \%)$ \\
\hline Marital status (DSW $\left.{ }^{\mathrm{a}}\right)$ & $5(2.0 \%)$ & $7(2.8 \%)$ \\
\hline Marital status (Unknown) & $0(0.0 \%)$ & $0(0.0 \%)$ \\
\hline Transmission route (Heterosexual) & 91 (36.4\%) & $66(26.4 \%)$ \\
\hline Marital status (Single) & $22(8.8 \%)$ & $13(5.2 \%)$ \\
\hline Marital status (Married) & $56(22.4 \%)$ & $27(10.8 \%)$ \\
\hline Marital status (DSW ${ }^{\mathrm{a}}$ ) & $13(5.2 \%)$ & $25(10.0 \%)$ \\
\hline Marital status(Unknown) & $0(0.0 \%)$ & $1(0.4 \%)$ \\
\hline Transmission route (Others) & $15(6.0 \%)$ & $3(1.2 \%)$ \\
\hline Marital status (Single) & $8(3.2 \%)$ & $1(0.4 \%)$ \\
\hline Marital status (Married) & $3(1.2 \%)$ & $1(0.4 \%)$ \\
\hline Marital status (DSWª) & $4(1.6 \%)$ & $1(0.4 \%)$ \\
\hline Marital status (Unknown) & $0(0.0 \%)$ & $0(0.0 \%)$ \\
\hline
\end{tabular}

${ }^{a} D S W$ Divorced, separated, or widowed 
variants from 440 participants were finally determined. 160 strains were CRF07_BC (36.4\%), 150 strains were CRF01_AE (34.1\%), 45 strains were CRF55_01B (10.2\%), 28 strains were subtype B (6.4\%), 15 strains were CRF08_ BC (3.4\%), 4 strains were CRF59_01B (0.9\%), 3 strains were subtype $C(0.7 \%), 1$ strain was subtype $D(0.2 \%), 1$ strain was CRF68_01B (0.2\%), 1 strain was CRF67_01B $(0.2 \%)$, and 32 strains were unique recombinant forms (URFs, 7.3\%) (Fig. 1). The subtyping proportions in years 2013 and 2015 are summarized in Additional file 2: Table S2. The subtypes were further assessed using phylogenetic trees that were constructed using the sequences and the reference sequences representing subtypes A-D, F-H, J, K, and CRFs epidemic in China (including CRF01_AE, CRF07_BC, CRF08_BC, CRF31_BC, CRF55_01B, CRF57_ BC, CRF59_01B, CRF61_BC, CRF62_BC, CRF64_BC, CRF65_cpx, CRF67_01B, and CRF68_01B), with subtype $\mathrm{O}$ as outgroup (http://www.hiv.lanl.gov, the reference information is summarized in Additional file 1: Table S1) (Fig. 4). By comparing the proportions of each subtype in 2013 and 2015, we found that the predominant strains shifted from CRF01_AE (36.10\% vs. 31.96\%) to CRF07_BC (34.84\% vs. $37.90 \%)$. However, no statistical significance was observed (Fig. 1).

A total of 32 URFs were identified in Shenzhen City among newly reported HIV-positive individuals. The compositions of the genomes of each URF were further determined. Four patterns of genomes of URFs were distinguished, including B/C/CRF01_AE, C/CRF01_AE, B/ CRF01_AE, and $\mathrm{B} / \mathrm{C}$ recombinant forms. Considering that CRF01_AE and CRF07_BC are the predominant strains prevalent in Shenzhen City, it is unsurprising to find that the genomes of the highest proportion of URFs comprised B, C, and CRF01_AE (Fig. 1).

\section{Phylogenetic analysis of HIV sequences}

To estimate the diversity of the sequences in this study within their respective subtypes, we calculated the genetic distances of different subtype sequences using the Kimura 2-parameter model based on the gag and pol genes (Table 3). The largest mean genetic distance was found in subtype B $(8.6 \% \pm 0.4 \%)$ within the gag gene. Relatively smaller mean genetic distances were found in CRF55_01B (3.9\% $\pm 0.2 \%)$ and CRF07_BC $(0.4 \% \pm 0.2 \%)$.
To explore the phylogenetic relationship of HIV strains with strains prevalent in other areas of China, we further aligned our sequences of CRF01_AE and CRF07_ $\mathrm{BC}$ subtypes with references from the whole country. In the ML trees, the strains from Shenzhen were distributed in the phylogenetic trees equally, and no significant local cluster within the Shenzhen area could be identified (Fig. 2). The results revealed a close relationship between the HIV prevalence in Shenzhen and other areas. Therefore, the floating population in Shenzhen City might serve as a bridge for HIV epidemics that have spread to different areas in China.

Significant differences in topology structures of phylogenetic trees constructed with CRF01_AE and CRF07_ $\mathrm{BC}$ sequences were observed. In the CRF07_BC tree, no large clusters containing more than 10 sequences were observed. However, in the CRF01_AE tree, at least four clusters were distinguished, with high bootstrap values. (Fig. 3). Cluster I (55.26\%) is the most popular group, followed by clusters III (21.05\%), IV (14.91\%), and II (8.77\%). Comparing the percentages of each cluster in 2013 and 2015 indicated that the percentage of cluster I increased from 50.91 to $59.32 \%$, whereas cluster III decreased from 29.09 to $13.56 \%$. Although no statistical significance was observed, the change in the ratio predict a change in CRF01_AE epidemic tendency in Shenzhen City. Further analysis of demographic information showed that the compositions of transmission routes of each cluster were significantly different (Fig. 3). Cluster I was mainly composed of sequences from MSMs (73.02\%). Cluster II contained only strains obtained from heterosexual transmitted individuals (100\%). Cluster III was mainly composed of strains from heterosexual transmission (83.33\%) and IDUs (12.50\%). Cluster IV was mainly composed of strains from heterosexual transmissions (58.82\%) and MSMs (35.29\%). Since the single, young MSMs became a high risk population for HIV infection in Shenzhen City, we further checked whether there were transmissions primarily confined to the population. The location of the sequences obtained from them was labeled in the phylogenetic tree (Fig. 4). No epidemic cluster that mainly comprised sequences from single young MSMs could be identified, indicating that there were no HIV strains that were confined to the specific population.

Table 3 Genetic distances among sequences belonging to different subtypes

\begin{tabular}{llllllll}
\hline Gene & \multicolumn{6}{l}{ Genetic distance $($ mean \pm SD $)$} \\
\cline { 2 - 7 } & CRF01_AE & CRF07_BC & CRF55_01B & B & CRF59_01B & CRF08_BC & C \\
\hline gag $(n=313)$ & $0.065 \pm 0.003$ & $0.040 \pm 0.002$ & $0.039 \pm 0.002$ & $0.086 \pm 0.004$ & $0.056 \pm 0.006$ & $0.052 \pm 0.004$ & $0.067 \pm 0.007$ \\
& $(n=128)$ & $(n=113)$ & $(n=37)$ & $(n=21)$ & $(n=2)$ & $(n=10)$ & $(n=2)$ \\
pol $(n=375)^{\mathrm{a}}$ & $0.054 \pm 0.003$ & $0.027 \pm 0.002$ & $0.023 \pm 0.002$ & $0.066 \pm 0.004$ & $0.030 \pm 0.004$ & $0.039 \pm 0.003$ & $0.048 \pm 0.005$ \\
& $(n=131)$ & $(n=152)$ & $(n=45)$ & $(n=26)$ & $(n=4)$ & $(n=14)$ & $(n=3)$ \\
\hline
\end{tabular}

a Only subtypes containing 3 or more sequences were included 

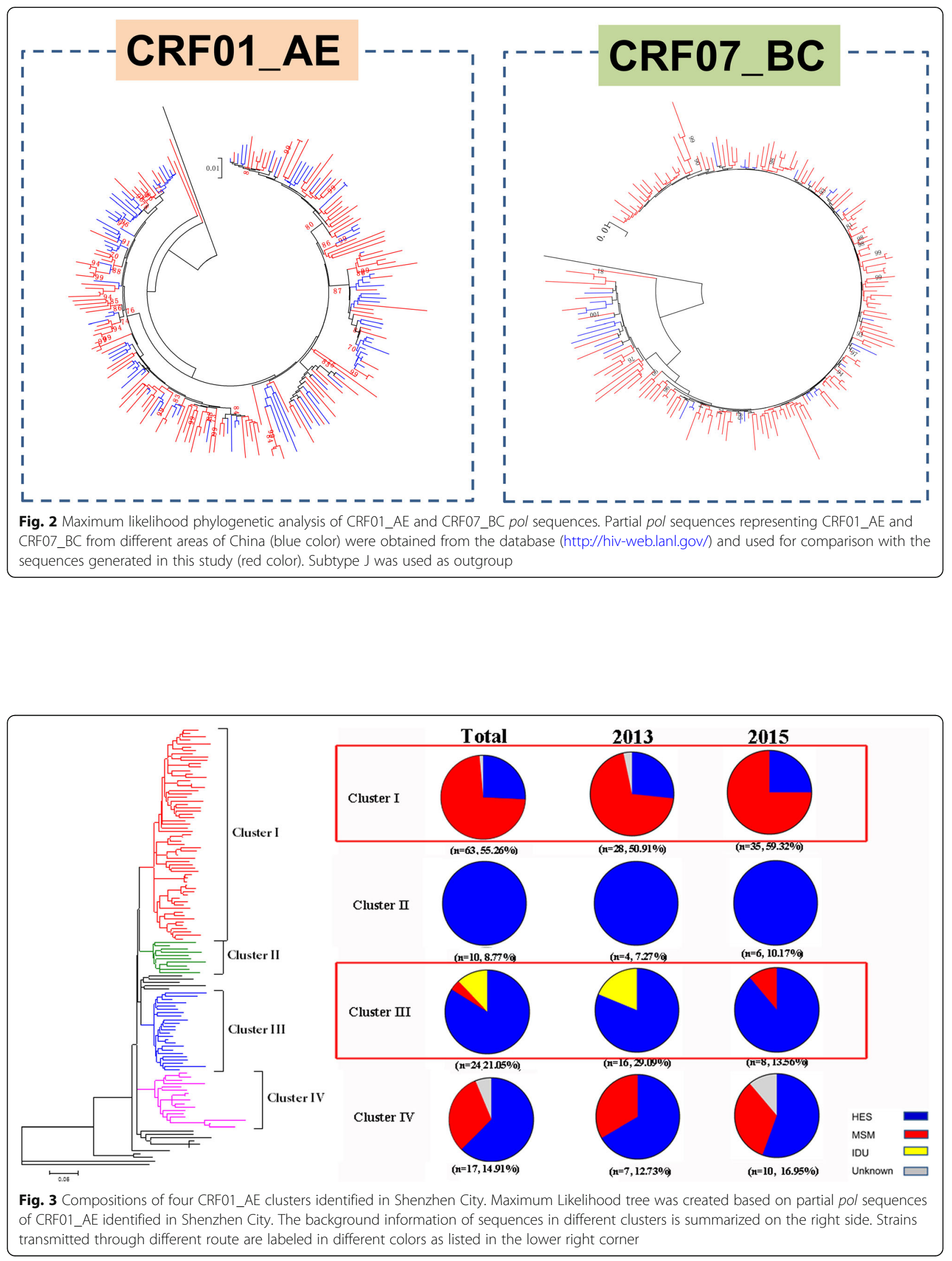


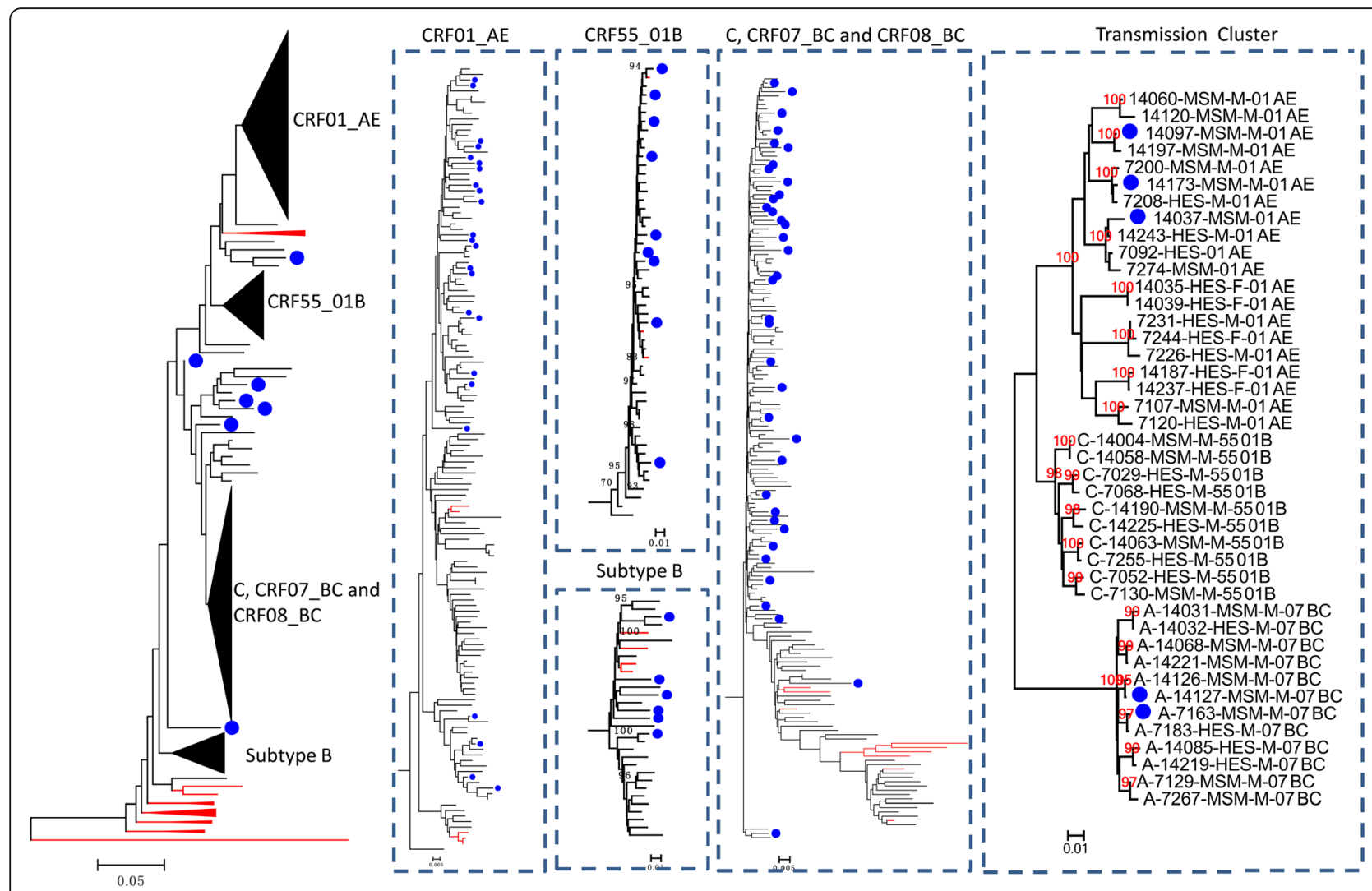

Fig. 4 Phylogenetic analysis of HIV strains identified in Shenzhen city. Neighbor-joining tree was created with partial pol genes of our sequences and the reference sequences of subtypes A-D, F-H, J, K, CRF01_AE, CRF07_BC, CRF08_BC, CRF55_01B, CRF59_01B, CRF67_01B, CRF68_01B and group $O$ (http://hiv-web.lanl. gov/). Each reference sequence is indicated by a red line. Sequences from single, young MSMs are labeled with a blue dot

To understand the HIV transmission dynamics of young, single MSMs and other populations in Shenzhen City, we further evaluated the phylogenetic clustering and determined demographic, behavioral, and geographic characteristics of persons in transmission clusters that included young, single MSMs [22]. Clusters with bootstrap values higher than $95 \%$ and mean genetic distances less than 0.015 were selected as transmission clusters or pairs. A total of 20 transmission clusters or pairs were identified, which contained 44 sequences. Among all of the sequences, five sequences were from young, single MSMs. No transmission cluster or pair only containing young, single MSMs was identified. All of the young single MSMs were involved in clusters containing old MSMs or heterosexually transmitted individuals (Fig. 4). The viral diversity in the MSM population over time is presented in Additional file 2: Table S3.

\section{Drug resistance analysis}

The $417 \mathrm{pol}$ genes obtained in the study were submitted online to screen for transmitted drug resistance mutations as described in Materials and Methods. Drug resistance mutations were identified in two samples from
2013 (0.4) and seven samples from 2015 (2.8\%), which were both below the WHO HIVDR Threshold Surveillance threshold of 5\%, indicating that the local ART programs were functioning well. A total of nine mutations (three NRTI-resistant mutations, two NNRTI-resistant mutations, and four PI-resistant mutations) were identified and are listed in Table 4.

\section{Discussion}

We compared the characterizations of newly reported HIV-positive cases in Shenzhen City between 2013 and 2015. A shift in HIV-positive populations was observed. Single, young MSMs were responsible for the observed higher number of HIV infections in 2015 compared to those in 2013. No HIV epidemic confined to single, young MSMs was identified. Close phylogenetic relationships among HIV strains between Shenzhen and other areas in China were observed both in 2013 and 2015. The results are important and helpful in terms of developing accurate preventive strategies for HIV control in Shenzhen City. It also highlights the importance of HIV surveillance, considering the rapid shifts in HIV-positive populations. 
Table 4 Drug resistant mutations detected in newly reported HIV infected individuals in Shenzhen

\begin{tabular}{|c|c|c|c|c|c|c|}
\hline \multirow[t]{2}{*}{ ID } & \multirow[t]{2}{*}{ Gender } & \multirow{2}{*}{$\begin{array}{l}\text { Transmission } \\
\text { route }\end{array}$} & \multirow{2}{*}{$\begin{array}{l}\text { Viral } \\
\text { Subtype }\end{array}$} & \multicolumn{3}{|c|}{ Drug-resistant mutations } \\
\hline & & & & PIs & NRTIs & NNRTIS \\
\hline 7137 & M & HES & CRF01_AE & L76 V & - & - \\
\hline 7142 & M & HES & CRF01_AE & - & $\llcorner 41 ।$ & - \\
\hline 14,003 & $\mathrm{~F}$ & HES & B & - & - & $\mathrm{K} 101 \mathrm{E}$ \\
\hline 14,037 & M & MSM & CRF01_AE & V82ILV & - & - \\
\hline 14,084 & M & HES & CRF59_01B & F53 L & - & - \\
\hline 14,139 & $\mathrm{~F}$ & HES & CRF01_AE & - & - & L100I \\
\hline 14,146 & M & HES & CRF07_BC & N83D & - & - \\
\hline 14,173 & M & MSM & CRF01_AE & - & L74I & - \\
\hline 14,179 & M & HES & CRF01_AE & - & V75 M & - \\
\hline
\end{tabular}

PIs protease inhibitors, NRT/s nucleoside reverse transcriptase inhibitors, NNRT/s non-nucleoside reverse transcriptase inhibitors

Public awareness, early initiation, and adherence to highly active antiretroviral treatment in index cases, and effective prevention strategies are all known to be important in the control of HIV epidemics. However, the vulnerable groups need to be specified as targets for intervention. It will be more cost-effective to use the limited HIV/AIDS resources available for target groups of people who are at the highest risk of infection. In this study, by comparing the characterizations of newly reported HIV-positive cases in Shenzhen City between 2013 and 2015, we have found that the population with the highest risk of HIV infection had changed. The proportion of single, young MSMs had significantly increased, underscoring the need to evaluate and intensify prevention efforts for this population.

Explore the origin of HIV epidemics in the target population is important for understanding the transmission dynamics, which in turn may provide clues for behavior intervention. Therefore, we further tested the relationship between HIV strains in epidemics in single, young MSMs and other populations. No epidemic or transmission clusters could be identified in single, young MSMs, indicating that there were no close connections among them. More interconnections might happen between single, young MSMs and old MSMs or heterosexually transmitted populations. Behavioral intervention confined only to single, young MSMs may be insufficient in controlling further transmissions. This undoubtedly will make it more difficult for HIV prevention in Shenzhen City. More comprehensive studies are necessary to distinguish individuals connected to the population.

Multiple subtypes of HIV were found in Shenzhen City. We identified 11 HIV subtypes or CRFs in Shenzhen City. The emergence of many subtypes of HIV in the same city might be due to its high level of floating population. The floating population brings back and forth the HIV strains prevalent in different areas of
China. To some extent, the HIV epidemic in Shenzhen even can profile HIV epidemics in the whole country. In this study, we found that the ratio of CRF07_BC strains increased in 2015 compared to 2013. The tendency is in accordance with a previous study that focused on MSMs in Shenzhen City [23]. The results indicated that CRF07_BC might spread again in MSMs. In 2016, we conducted phylodynamic analysis of CRF07_BC strains prevalent in China, which showed that CRF07_BC had undergone a second rapid spread in China [24]. Therefore, further studies on the CRF07_BC epidemic in China are necessary. Multiple subtypes of HIV circulating in the same population always predict the emergence of recombinant. A high level of URFs (11.2\%) was observed in Shenzhen City. In 2015, CRF55_01B was reported to be formed in Shenzhen City and rapidly spread in MSMs outside Shenzhen City [25]. Therefore, surveying HIV URFs formed in Shenzhen City will be helpful in the prediction of the emergence of CRFs in China.

In 2003, the government of China launched the "Four Free and One Care" policy [26]. Since then, HAART has been available. However, along with the extensive use of HAART, HIV-1 drug resistance has become an issue in some areas of China. In some individuals receiving therapy, the ratio of HIV drug-resistant mutations was higher than $20 \%$ in some areas [27]. In this study, a low level of drug-resistant strains was found in Shenzhen City both in 2013 and 2015. However, more drugresistant variants were identified in 2015 compared to 2013, suggesting that the transmission of drug resistance has increased. Therefore, surveillance of HIV drug resistance is necessary in the region.

This study has some limitations. The two crosssectional studies are based on newly reported HIV positive cases collected in 2013 and 2015. The time period is only 2 years, which is relatively short for monitoring changes in large HIV-positive populations. However, some shifted characterizations of HIV-positive populations have been selected. If the time is long enough, then more changing characteristics on HIV epidemiological trends could be distinguished and may explain why no statistical significance was observed on HIV subtype distribution and even transmitted drug resistant mutations. In addition, although all of the participants were newly reported HIV positive cases, it is difficult to estimate the exact infection time. Hence, it was considered that the mean interval time for an individual to be detected after being infected was the same between 2013 and 2015 .

\section{Conclusions}

In conclusion, we observed that the proportion of single, young MSMs significantly increased in newly reported HIV-positive cases in Shenzhen City, which will provide 
additional information for HIV prevention and behavior intervention. The identification of many HIV subtypes circulating in Shenzhen City will provide more information that may be utilized for HIV vaccine design. Furthermore, the close phylogenetic relationship of HIV strains between Shenzhen and many other areas in China suggests that the Shenzhen HIV epidemic might be useful for the prediction of HIV prevalence in the entire country. This study also highlights the importance of more comparable cross-sectional studies, which will be cost-effective for surveillance of the HIV epidemic in China.

\section{Additional files}

Additional file 1: Table S1. Detailed information on the references used in this study. (XLSX $14 \mathrm{~kb}$ )

Additional file 2: Table S2. Subtyping proportions in 2013 and 2015. Table S3. Viral diversity in 2013 and 2015. (DOCX 15 kb)

\section{Abbreviations}

CRFs: Circulating recombinant forms; HIV: Human immunodeficiency virus; IDUs: Injecting drug users; jpHMM: jumping profile hidden Markov model; MSM: Men who have sex with men; URFs: Unique recombinant forms

\section{Acknowledgments}

We thank Chenxiao Lu for helping in the preparation of the manuscript.

\section{Authors' contributions}

Research design: $L L$ and HL Performed the analysis: DJ, YL, XW, $\sqcup$ and TG Contributed materials: JZ, LC, and CZ. Contributed to the composition of the manuscript: $L L, L J, J H, T L$, and $J$. All authors read and approved the final manuscript.

\section{Funding}

The National Natural Science Foundation of China (Nos. 81,773,493; 81,273,137; 81,573,211), the National Key S\&T Special Projects on Major Infectious Diseases (Grant No. 2012ZX10002), Beijing Municipal Science \& Technology Project (D141100000314001), and the State Key Laboratory of Pathogen and Biosecurity (Academy of Military Medical Science) supported this study.

\section{Availability of data and materials}

All of the data generated or analyzed during this study are included in this published article.

\section{Ethics approval and consent to participate}

This study and all of the relevant experiments have been approved by the Ethical Board of the Beijing Institute of Microbiology and Epidemiology. All of the patients provided written informed consent, and the data were analyzed anonymously.

\section{Consent for publication}

Not applicable.

\section{Competing interests}

The authors declare that they have no competing interests.

\section{Author details}

'Department of AIDS Research, State Key Laboratory of Pathogen and Biosecurity, Beijing Institute of Microbiology and Epidemiology, Beijing 100071, China. ${ }^{2}$ Shenzhen Center for Disease Control and Prevention, Shenzhen 518055, Guangdong, China.
Received: 15 November 2018 Accepted: 10 June 2019

Published online: 22 June 2019

\section{References}

1. Shang H, XU J, Han X, Spero Li J, Arledge KC, prevention ZLHIV. Bring safe sex to China. Nature. 2012:485:576-7.

2. Wang X, He X, Zhong P, Liu Y, Gui T, Jia D, et al. Phylodynamics of major CRF01_AE epidemic clusters circulating in mainland of China. Sci Rep. 2017; 7:6330.

3. Shi TX, Zhang BC, Li XF, XU JX, Wang N, Yu ZZ, et al. Study on the high risk behaviors related to AIDS among men who having sex with men in the floating population. Zhonghua Liu Xing Bing Xue Za Zhi. 2009;30:668-71.

4. Wang B, Li X, Stanton B, Fang X, Lin D, Mao R. HIV-related risk behaviors and history of sexually transmitted diseases among male migrants who patronize commercial sex in China. Sex Transm Dis. 2007:34:1-8.

5. He N. Sociodemographic characteristics, sexual behavior, and HIV risks of rural-to-urban migrants in China. Biosci Trends. 2007;1:72-80.

6. Li X, Zhang L, Stanton B, Fang X, Xiong Q, Lin D. HIV/AIDS-related sexual risk behaviors among rural residents in China: potential role of rural-tourban migration. AIDS Educ Prev. 2007;19:396-407.

7. Chow EP, Wilson DP, Zhang J, Jing J, Zhang L. Human immunodeficiency virus prevalence is increasing among men who have sex with men in China: findings from a review and meta-analysis. Sex Transm Dis. 2011;38: 845-57.

8. Tang W, Mahapatra T, Liu F, Fu G, Yang B, Tucker JD, et al. Burden of HIV and syphilis: a comparative evaluation between male sex workers and nonsex-worker men who have sex with men in urban China. PLoS One. 2015; 10:e0126604.

9. Zou X, Chow EP, Zhao P, Xu Y, Ling L, Zhang L. Rural-to-urban migrants are at high risk of sexually transmitted and viral hepatitis infections in China: a systematic review and meta-analysis. BMC Infect Dis. 2014;14:490.

10. Meng X, Wang L, Chan S, Reilly KH, Peng Z, Guo W, et al. Estimation and projection of the HIV epidemic trend among the migrant population in China. Biomed Environ Sci. 2011;24:343-8.

11. Zhang L, Chow EP, Jahn HJ, Kraemer A, Wilson DP. High HIV prevalence and risk of infection among rural-to-urban migrants in various migration stages in China: a systematic review and meta-analysis. Sex Transm Dis. 2013:40: 136-47.

12. Zhang L, Wang YJ, Wang BX, Yan JW, Wan YN, Wang J. Prevalence of HIV-1 subtypes among men who have sex with men in China: a systematic review. Int J STD AIDS. 2015;26:291-305.

13. Kouyos RD, von Wyl V, Yerly S, Boni J, Taffe P, Shah C, et al. Molecular epidemiology reveals long-term changes in HIV type 1 subtype B transmission in Switzerland. J Infect Dis. 2010:201:1488-97.

14. Holmes EC, Zhang LQ, Robertson P, Cleland A, Harvey E, Simmonds P, et al. The molecular epidemiology of human immunodeficiency virus type 1 in Edinburgh. J Infect Dis. 1995;171:45-53.

15. Brenner BG, Roger M, Routy JP, Moisi D, Ntemgwa M, Matte $C$, et al. High rates of forward transmission events after acute/early HIV-1 infection. J Infect Dis. 2007:195:951-9.

16. Yerly S, Vora S, Rizzardi P, Chave JP, Vernazza PL, Flepp M, et al. Acute HIV infection: impact on the spread of HIV and transmission of drug resistance. AIDS. 2001:15:2287-92

17. Yerly S, Junier T, Gayet-Ageron A, Amari EB, von Wyl V, Gunthard HF, et al. The impact of transmission clusters on primary drug resistance in newly diagnosed HIV-1 infection. AIDS. 2009;23:1415-23.

18. Smith DM, May SJ, Tweeten S, Drumright L, Pacold ME, Kosakovsky Pond SL, et al. A public health model for the molecular surveillance of HIV transmission in San Diego, California. AIDS. 2009:23:225-32.

19. Li L, Lu X, Li H, Chen L, Wang Z, Liu Y, et al. High genetic diversity of HIV-1 was found in men who have sex with men in Shijiazhuang, China. Infect Genet Evol. 2011;11:1487-92.

20. Li L, Liang S, Chen L, Liu W, Li H, Liu Y, et al. Genetic characterization of 13 subtype CRF01_AE near full-length genomes in Guangxi, China. AIDS Res Hum Retrovir. 2010;26:699-704.

21. Bennett DE, Camacho RJ, Otelea D, Kuritzkes DR, Fleury H, Kiuchi M, et al. Drug resistance mutations for surveillance of transmitted HIV-1 drugresistance: 2009 update. PLoS One. 2009:4:e4724.

22. Oster AM, Pieniazek D, Zhang X, Switzer WM, Ziebell RA, Mena LA, et al. Demographic but not geographic insularity in HIV transmission among young black MSM. AIDS. 2011;25:2157-65. 
23. Zhao J, Chen L, Chaillon A, Zheng C, Cai W, Yang Z, et al. The dynamics of the HIV epidemic among men who have sex with men (MSM) from 2005 to 2012 in Shenzhen, China. Sci Rep. 2016;6:28703.

24. Zhang M, Jia D, Li H, Gui T, Jia L, Wang X, et al. Phylodynamic analysis revealed that epidemic of CRF07_BC strain in men who have sex with men drove its second spreading wave in China. AIDS Res Hum Retrovir. 2017;33: 1065-9.

25. Zhao J, Cai W, Zheng C, Yang Z, Xin R, Li G, et al. Origin and outbreak of HIV-1 CRF55_01B among MSM in Shenzhen, China. J Acquir Immune Defic Syndr. 2014;66:e65-7.

26. Zhang FJ, Pan J, Yu L, Wen Y, Zhao Y. Current progress of China's free ART program. Cell Res. 2005;15:877-82.

27. Wang $Z$, Liang $Y, X$ in $T$. Investigation on curative effect and drug resistance of HIV/AIDS patients receiving HAART. J Med Forum. 2005;26:1-3.

\section{Publisher's Note}

Springer Nature remains neutral with regard to jurisdictional claims in published maps and institutional affiliations.

Ready to submit your research? Choose BMC and benefit from:

- fast, convenient online submission

- thorough peer review by experienced researchers in your field

- rapid publication on acceptance

- support for research data, including large and complex data types

- gold Open Access which fosters wider collaboration and increased citations

- maximum visibility for your research: over $100 \mathrm{M}$ website views per year

At $\mathrm{BMC}$, research is always in progress.

Learn more biomedcentral.com/submissions 